\title{
Pinning, retraction and terracing of evaporating droplets containing nanoparticles
}

\author{
R. V. Craster, O. K. Matar† and K. Sefianeł \\ Department of Mathematical and Statistical Sciences, \\ University of Alberta, \\ Edmonton, T6G 2G1, Canada \\ $\dagger$ Department of Chemical Engineering, \\ Imperial College London, \\ South Kensington Campus, \\ London, SW7 2AZ, U.K. \\ $\ddagger$ School of Engineering 85 Electronics, \\ University of Edinburgh, \\ Mayfield Rd., Edinburgh, EH9 3JL, UK
}

\begin{abstract}
We consider the dynamics of a slender, evaporating droplet containing nanoparticles. We use lubrication theory to derive a coupled system of equations that govern the film thickness and the concentration of nanoparticles. These equations account for capillarity, Marangoni stresses, evaporation and disjoining pressure; the nanoparticles-induced structural component of the disjoining pressure is also considered. Contact line singularities are avoided through the adsorption of ultrathin films wherein evaporation is suppressed by the disjoining pressure; a similar approach has recently been used by Ajaev [J. Fluid Mech., 528, 279-296, 2005] who has built on the previous work of Moosman and Homsy [J. Colloid Interface Sci., 73, 212-223, 1980]. The results of our numerical simulations indicate that, depending on the value of system parameters, the droplet exhibits a variety of different behaviours, which include spreading, evaporation-driven retraction, contact line pinning, and 'terrace'-formation.
\end{abstract}




\section{INTRODUCTION}

The spreading of fluids on the surface of solids is dependent on the interfacial tensions and the film energy, an integral of the disjoining pressure over the film thickness. The disjoining pressure comprises van der Waals forces, electrostatic interactions and steric and structural effects $[1,2]$. The latter effects can play a key role in the spreading of nanofluids. This is due to particle confinement between the liquid-solid and air-liquid interfaces in the vicinity of the contact line. Decreasing the separation between the two interfaces leads to their entropically-driven attraction, which drives phase separation in colloidal dispersions [1,3-8]. The structural component of the disjoining pressure is oscillatory in the direction normal to the interacting surfaces. Such oscillatory structural interactions also arise in thin liquid layers sandwiched between two smooth solid walls even in the absence of nanoparticles [6]; in this case, they are referred to as "solvation" forces. The presence of these forces has been shown to give rise to 'stepwise' thinning of foams and colloidal dispersions [9-18], with particle layering between $5 \mathrm{~nm}$ and $2 \mu \mathrm{m}[6,12]$, and the 'terraced' spreading of nanodroplets $[19,20]$.

Structural forces can have a much longer range than van der Waals interactions $[8,11]$ leading to an increase in the spreading rate of thin films [21]. These effects have been examined within the context of detergency and the promotion of oil droplet detachment from solids by surfactant solutions $[8,11,21-23]$. The equilibrium meniscus shape near the contact line of a drop laden with nanoparticles has been calculated recently by $[8,23]$ who accounted for the structural disjoining pressure component for an oil-aqueous phase system. An analytical expression for this component was used $[8,23]$ based on the Percus-Yevick theory, which treats the confining surfaces and the nanoparticles as hard-spheres. This expression accounts for the oscillations in this component as a function of the film thickness, due to particle layering, followed by exponential decay at large thicknesses; when the film thickness equals the particle diameter, this expression includes a term, which ensures that

the Percus-Yevick predictions are consistent with exact statistical mechanical results [7]. The results of these studies showed that an increase in contact line displacement resulted from a rise in the concentration of nanoparticles, and a decrease in their diameter and the degree of their polydispersity.

More recently, Matar et al. [24], examined the effect of the structural component of the 
disjoining pressure on the spreading dynamics of a droplet. They used lubrication theory to derive a pair of coupled evolution equations for the thin film thickness and nanoparticle concentration; the approach of Trokhymchuk et al. and Chengara et al. [8, 23] was followed to model the presence of structural effects. Their results indicated the development of a 'step' near the contact line, which became more pronounced with increasing particle concentration. The results of simulations showing terracing of droplets were also reported.

A number of studies have also examined the evaporation of thin films and droplets in the absence [25-33] and presence of particles [34-42]. The latter works have demonstrated the development of rings in the drying of coffee drops and films containing latex particles [35-37], the formation of rings [43-45] and tree-like structures [46] in nanoparticulate thin evaporating films of binary mixtures of nitrocellulose in amyl acetate and hexadecylamine in hexane, complex stain morphologies in crystallising droplets [38], stick-slip motion [41], and fingering phenomena in dewetting films of nanofluids [40, 42]. (There are also related studies that examine the patterns formed during the evaporation of drops and films of polymer solutions [47-49].) Yet, in spite of this large number of studies, to the best of our knowledge, none of these has modelled the flow dynamics in the simultaneous presence of structural forces and phase change effects. The combination of the effects may be significant: it is well known that the evaporative flux is largest near the contact line of a drop [30, 35, 36]; evaporation from this region increases particle concentration, thereby increasing the relative significance of the structural component. Although this may promote spreading [21], an increase in the particle concentration also leads to an increase in viscosity, which acts to retard the spreading. Thus, careful modelling of the dynamics is required in order to determine the outcome of the competition between these phenomena; this is the main aim of our paper.

The rest of the paper is organised as follows. In section II, we provide details of the model derivation. Here, we build on the work of Chengara et al. [23], Ajaev [30] and Matar et al. [24] and use lubrication theory to derive coupled evolution equations for the film thickness and particle concentration, which accounts for evaporation and disjoining pressure effects. The results of our numerical simulations are presented in section III and concluding remarks are provided in section IV. 


\section{FORMULATION}

\section{A. Governing equations}

We consider the dynamics of a thin, evaporating droplet, which contains monodisperse nanoparticles of diameter $d$. The characteristic thickness and length of this droplet are $\mathcal{H}$ and $\mathcal{L}$, respectively. A rectangular coordinate system, $(x, z)$, is used to model the twodimensional film flow, in which $x$ and $z$ denote the horizontal and vertical coordinates, respectively. The drop is bounded by a solid wall at $z=0$ and by an inviscid gas from above. The gas-liquid interface is located at $z=h(x, t)$, which has a constant surface tension, $\sigma$. The velocity field is denoted by $\mathbf{u}=(u, w)$ wherein $u$ and $w$ represent the horizontal and vertical velocity components, respectively. The drop viscosity is dependent on the volume fraction of the particles, $\phi$, through a Krieger-Dougherty-type relation, $\mu=\mu_{0}\left(1-\phi / \phi_{m}\right)^{-2}$, where $\phi_{m}$ is the volume fraction at close packing.

The dynamics are modelled using lubrication theory, [26, 50], by assuming that $\epsilon \equiv$ $\mathcal{H} / \mathcal{L} \ll 1$. The mass, momentum and energy conservation equations are then given by [30]

$$
u_{x}+w_{z}=0, \quad p_{x}=\left(\mu(\phi) u_{z}\right)_{z}, \quad p_{z}=0, \quad T_{z z}=0,
$$

where $p$ and $T$ denote the pressure and temperature, respectively, and gravitational forces have been neglected; we also neglect convective heat transfer effects and assume conduction to be dominant. Equations (1) are solved subject to no-slip and no-penetration conditions at the wall, $u=w=0$ at $z=0$; we also assume the wall to be highly conducting and set $T=T_{w}$ at $z=0$. Normal and tangential stress conditions are applied at $z=h$ in addition to kinematic and thermal flux conditions; following the application of the lubrication approximation, these conditions are respectively given by

$$
p=p_{v}-\sigma h_{x x}-\Pi, \quad \mu u_{z}=\sigma_{x}+h_{x} \sigma_{z}, \quad h_{t}+u h_{x}-w+\frac{J}{\rho_{l}}=0, \quad \Delta H_{v} J=-\lambda T_{z},
$$

where $\Pi$ represents the disjoining pressure, $J$ denotes the evaporative flux, $\rho_{l}$ is the fluid density and $p_{v}$ is the vapour pressure; $\Delta H_{v}$ and $\lambda$ are the latent heat of vapourisation and (constant) thermal conductivity, respectively. The surface tension, $\sigma$, depends on temperature linearly through $\sigma=\sigma_{0}-\gamma\left(T-T_{s}\right)$, where $\gamma \equiv-d \sigma / d T$ and $\sigma_{0}$ denotes the value of the surface tension at the equilibrium saturation temperature, $T_{s}$. In Eqs. (2), we have 
neglected vapour recoil effects in the normal stress balance, and kinetic energy effects and the thermal conductivity of the vapour phase in the thermal flux condition.

In order to model evaporative effects, the following non-equilibrium interfacial condition is applied $[30,51,52]$

$$
\frac{p_{v e}}{p_{v}}-1=\frac{\sigma}{\rho_{l} R T_{s}}\left(p-p_{v}\right)+\frac{\Delta H_{v}}{R T_{s}}\left(\frac{T_{i}}{T_{s}}-1\right),
$$

where $T_{i}$ corresponds to the interfacial temperature, $R$ is the gas constant per unit volume, and $p_{v e}$ is the equilibrium vapour pressure. The following equilibrium relation is used for the saturation temperature, $T_{s},[30,51,52]$

$$
T_{s}=\frac{2 \pi}{R}\left(\frac{J}{\rho_{v}\left(\frac{p_{v e}}{p_{v}}-1\right)}\right)^{2} .
$$

The intermolecular interactions are modelled using a combination of oscillatory structural disjoining pressures and van der Waals interactions: $\Pi=\Pi_{v w}+\Pi_{o s}$, wherein $\Pi_{o s}$ denotes the structural component given by [6]

$$
\begin{aligned}
& \Pi_{o s}=P \cos \left(\frac{2 \pi h}{d_{1}}\right) \exp \left(\frac{d^{3}}{d_{1}^{2} d_{2}}-\frac{h}{d_{2}}\right), \quad h \geq d, \\
& \Pi_{o s}=-P, \quad 0<h<d,
\end{aligned}
$$

and van der Waals interactions, $\Pi_{v w}$, are expressed by

$$
\Pi_{v w}=\frac{A}{6 \pi h^{3}}
$$

Alternative structural disjoining pressures $[8,23]$ are available and were used by the present authors in previous work [24]. The expression for $\Pi_{o s}$ in Eq. (5) is simpler in form and encompasses the decaying oscillatory behaviour in $h$, which is an essential ingredient of the model. In Eq. (5), $d$ is the particle diameter; $d_{1}=d f_{1}(\phi)$ and $d_{2}=d f_{2}(\phi)$ in which

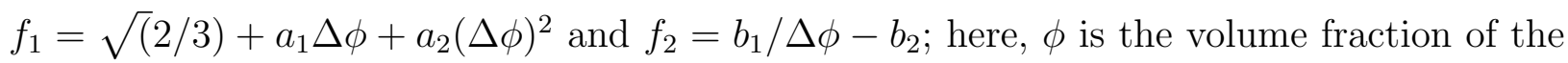
particles, $\Delta \phi=\pi / 3 \sqrt{2}-\phi, a_{1}=0.23728, a_{2}=0.63300, b_{1}=0.48663$ and $b_{2}=0.42032[6]$. Equation (5) comprises an oscillatory, structural disjoining pressure component for $h>d$, which arises when interactions of surfaces separated by model fluids are considered using statistical mechanical arguments [53]. For $h<d$, depletion forces are present, represented by $P=\rho k T f_{3}(\phi)$ and for all $h$ van der Waals forces are present and represented by $A / 6 \pi h^{3}$; additionally, $A$ is the Hamaker constant, $\rho=6 \phi / \pi d^{3}$ denotes the number density of the 
particles, $k$ is the Boltzmann constant and $f_{3}=\left(1+\phi+\phi^{2}-\phi^{3}\right) /(1-\phi)^{3}$; electrostatic contributions have been neglected.

The dynamics of the particle concentration, $c$, are governed by a standard convectivediffusion equation, which is expressed by

$$
c_{t}+u c_{x}+w c_{z}=\mathcal{D}\left(c_{x x}+c_{z z}\right),
$$

in which $\mathcal{D}$ is the diffusion coefficient of the nanoparticles. Solutions of Eq. (7) are sought subject to the following boundary conditions

$$
\begin{aligned}
c_{z} & =0, \quad z=0, \\
\mathcal{D}(\mathbf{n} \cdot \nabla) c & =c\left(\mathbf{u}-\mathbf{u}_{s}\right) \cdot \mathbf{n},
\end{aligned}
$$

where $\mathbf{u}_{s}$ denotes the velocity of the interface. These conditions correspond to no-flux at the wall and a diffusive flux at $z=h$ due to concentration variations associated with evaporation. Note that $\phi$ is taken to be related to $c$ by $\phi=\phi_{m}\left(c / \rho_{p}\right) /\left[1+\left(c / \rho_{p}\right)\right]$ in which $\rho_{p}$ is the density of individual particles (see appendix). Next, we exploit the slenderness of the drop to derive evolution equations that govern its dynamics.

\section{B. Scaling and cross-sectional averaging}

We render the governing equations dimensionless using the following scalings in which the tildes denote dimensionless variables

$$
\begin{gathered}
x=\mathcal{L} \tilde{x}, \quad(z, h)=\mathrm{Ca}^{1 / 3} \mathcal{L}(\tilde{z}, \tilde{h}), \quad t=(\mathcal{L} / \mathcal{U}) \tilde{t}, \\
(u, w)=\mathcal{U}\left(\tilde{u}, \mathrm{Ca}^{1 / 3} \tilde{w}\right), \quad(p, P, \Pi)=\left(\sigma \mathrm{Ca}^{1 / 3} / \mathcal{L}\right)(\tilde{p}, \tilde{P}, \tilde{\Pi}), \\
T=T_{s}+\mathrm{Ca}^{2 / 3} T_{s} \tilde{T}, \quad c=c_{\rho} \tilde{c}, \quad \phi=\phi_{m} \tilde{\phi}, \quad J=\rho_{l} \mathcal{U} \mathrm{Ca}^{1 / 3} \tilde{J}, \quad \mu=\mu_{0} \tilde{\mu} .
\end{gathered}
$$

Here, $\phi_{m}=0.664$ denotes the volume fraction and in terms of $\tilde{c}$, the normalised particle volume fraction, $\tilde{\phi}$, can be expressed as $\tilde{\phi}=\tilde{c} /[1+\tilde{c}]$. The capillary number, $\mathrm{Ca} \equiv \mu_{0} \mathcal{U} / \sigma_{0} \ll$ 1 , is a small parameter, where $\mathcal{U} \equiv \lambda T_{s} / \rho_{l} \Delta H_{v} \mathcal{L}$ represents a characteristic velocity. Note that $\mathrm{Ca}^{1 / 3}=\mathcal{H} / \mathcal{L} \ll 1$, which is the droplet aspect ratio. The tildes are henceforth suppressed. 
The dimensionless convective diffusion equation governing the evolution of $c$ is then given by

$$
c_{t}+u c_{x}+w c_{z}=\frac{1}{\mathrm{Ca}^{2 / 3} \mathrm{Pe}}\left(c_{z z}+\mathrm{Ca}^{2 / 3} c_{x x}\right),
$$

where $\mathrm{Pe} \equiv \mathcal{U L} / \mathcal{D}$ is a Peclet number characterising the importance of convective versus diffusive particle transport. The following boundary conditions are imposed on the solutions of Eq. (10)

$$
\begin{aligned}
c_{z} & =0, \quad \text { at } z=0, \\
-\mathrm{Ca}^{2 / 3} h_{x} c_{x}+c_{z} & =\mathrm{Ca}^{2 / 3} \operatorname{Pe} c\left[-h_{x}\left(u-u_{s}\right)+w-w_{s}\right] \quad \text { at } \quad z=h .
\end{aligned}
$$

We shall work in the rapid vertical diffusion limit and assume $\mathrm{Ca}^{2 / 3} \mathrm{Pe} \ll 1$ so that we can substitute $c(x, z, t)=c_{0}(x, t)+\mathrm{Ca}^{2 / 3} \mathrm{Pe} c_{1}(x, z, t)$ into Eq. (10) [54]:

$$
c_{0 t}+u c_{0 x}=\frac{1}{\mathrm{Pe}} c_{0 x x}+c_{1 z z}+O\left(\mathrm{Ca}^{2 / 3} \mathrm{Pe}\right) .
$$

A similar substitution into Eq. (11) yields

$$
\begin{aligned}
& c_{1 z}=0, \quad \text { at } \quad z=0, \\
& c_{1 z}=\frac{h_{x} c_{0 x}}{\mathrm{Pe}}+c_{0} J, \quad \text { at } \quad z=h,
\end{aligned}
$$

where use was also made of Eq. (A1). Cross-sectional averaging of Eq. (12) and application of Eqs. (13) yields

$$
c_{0 t}+\bar{u} c_{0 x}=\frac{1}{h \mathrm{Pe}}\left(h c_{0 x}\right)_{x}+\frac{c_{0} J}{h},
$$

where $\bar{u}=(1 / h) \int_{0}^{h} u d z$ is the average droplet velocity, and the cross-sectional average of $c_{1}(x, z, t)$ is taken to be zero so that $(1 / h) \int_{0}^{h} c_{1} d z=0$. The normalised particle volume fraction at leading order in $\mathrm{Ca}^{2 / 3} \mathrm{Pe}$ is $\phi_{0}=c_{0} /\left[1+c_{0}\right]$.

The dimensionless governing equations remain unchanged from Eqs. (1), while the dimensionless interfacial conditions are expressed by

$$
p=p_{v}-h_{x x}-\Pi, \quad \mu u_{z}=-\mathrm{Ma}\left(T_{x}+h_{x} T_{z}\right), \quad h_{t}+u h_{x}-w+J=0, \quad J=-T_{z},
$$

where Ma $\equiv \gamma T_{s} / \sigma_{0}$ is a Marangoni number. Note that in the normal stress condition, only the leading order contribution of the dimensionless capillary term has been retained: $\left(1-\mathrm{Ca}^{2 / 3} \mathrm{MaT}\right) h_{x x}=h_{x x}+O\left(\mathrm{Ca}^{2 / 3}\right)$; that is, Marangoni effects on capillarity have been 
neglected at leading order. The dimensionless expression for $\Pi$ is $\Pi_{o s}+\Pi_{v w}$ where

$$
\begin{array}{ll}
\Pi_{o s}=P \cos \left(\frac{2 \pi h}{\varepsilon f_{1}\left(\phi_{0}\right)}\right) \exp \left(\frac{1}{f_{1}^{2}\left(\phi_{0}\right) f_{2}\left(\phi_{0}\right)}-\frac{h / \varepsilon}{f_{2}\left(\phi_{0}\right)}\right), & h \geq \varepsilon, \\
\Pi_{o s}=-P, & 0<h<\varepsilon,
\end{array}
$$

where $\varepsilon \equiv d / \mathrm{Ca}^{1 / 3} \mathcal{L}$ is a dimensionless particle diameter and $P=(6 / \pi) \mathcal{S} \phi_{0} f_{3}\left(\phi_{0}\right)(1+$ $\left.\mathrm{Ca}^{2 / 3} T\right)=(6 / \pi) \mathcal{S} \phi_{0} f_{3}\left(\phi_{0}\right)+O\left(\mathrm{Ca}^{2 / 3}\right)$ in which $\mathcal{S} \equiv k T_{s} \mathcal{L} / \sigma_{0} d^{3} \mathrm{Ca}^{1 / 3}$, a dimensionless parameter that represents the relative significance of structural disjoining pressure effects; here, the effect of thermal variations on $P$ have been neglected at leading order. The van der Waals component of the disjoining pressure is also scaled so

$$
\Pi_{v w}=\frac{\mathcal{A}}{h^{3}}
$$

where $\mathcal{A}$ is a rescaling of the Hamaker constant to

$$
\mathcal{A}=\frac{A}{6 \pi \sigma_{0} \mathcal{H}^{2} \epsilon^{2}} \text {. }
$$

The dimensionless constitutive relation for $J$, which results from Eqs. (3) and (4) is given by

$$
K J=\Delta\left(p-p_{v}\right)+T_{i}
$$

where $T_{i}$ is the interfacial temperature; $K \equiv \rho_{l} \mathcal{U} \sqrt{2 \pi R T_{s}} / \rho_{v} \mathrm{Ca}^{1 / 3} \Delta H_{v}$ and $\Delta \equiv$ $\sigma_{0} / \rho_{l} \mathcal{L} \Delta H_{v} \mathrm{Ca}^{1 / 3}$ are parameters that characterise the importance of interfacial kinetic effects and of variations in the pressure on the phase-change interfacial temperature [30]. The conditions at $z=0$ are given by

$$
u=w=0, \quad T=\frac{T_{w}-T_{s}}{T_{s} \mathrm{Ca}^{2 / 3}} \equiv \Theta,
$$

where $\Theta$ represents a dimensionless difference between the wall temperature and the equilibrium saturation temperature.

The leading order temperature equation, $T_{z z}=0$, is integrated twice and the temperature conditions in Eqs. (15) and (19) are imposed, which yields

$$
T=\Theta-z J
$$

The $x$-component of the momentum conservation equation is also readily integrated and the tangential stress and no-slip boundary conditions in Eqs. (15) and (19) applied (after making use of Eq. (20)) to yield the following expression for $u$

$$
u=\frac{1}{\mu\left(\phi_{0}\right)}\left[\operatorname{Maz}(h J)_{x}+p_{x}\left(\frac{z^{2}}{2}-z h\right)\right],
$$


whence $\bar{u}$ is readily obtained:

$$
\bar{u}=\frac{1}{\mu\left(\phi_{0}\right)}\left[\operatorname{Ma} \frac{h}{2}(h J)_{x}-\frac{h^{2}}{3} p_{x}\right]
$$

Since $p_{z}=0$, the pressure is given by the normal stress condition in Eq. (15),

$$
p=p_{v}-h_{x x}-\Pi \text {. }
$$

Substitution of Eq. (20) and (23) into Eq. (18) yields an expression for $J$ :

$$
J=\frac{\Theta-\Delta\left(h_{x x}+\Pi\right)}{K+h} .
$$

The kinematic boundary condition in Eq. (15) can be re-expressed as $h_{t}+(\bar{u} h)_{x}+J=0$. Substitution of Eq. (22) into this equation and Eq. (14) finally yields evolution equations for $h$ and $c_{0}$, respectively:

$$
\begin{gathered}
h_{t}+\left(\frac{1}{\mu\left(\phi_{0}\right)}\left[\operatorname{Ma} \frac{h^{2}}{2}(h J)_{x}-\frac{h^{3}}{3} p_{x}\right]\right)_{x}+J=0, \\
c_{0 t}+\frac{1}{\mu\left(\phi_{0}\right)}\left[\operatorname{Ma} \frac{h}{2}(h J)_{x}-\frac{h^{2}}{3} p_{x}\right] c_{0 x}=\frac{1}{h \mathrm{Pe}}\left(h c_{0 x}\right)_{x}+\frac{c_{0} J}{h},
\end{gathered}
$$

where $p$ and $J$ are given by Eqs. (23) and (24), respectively, and $\mu=\left(1-\phi_{0}\right)^{-2}$; the ' 0 ' subscript is suppressed henceforth. Estimates of the magnitudes of the dimensionless groups for the structural disjoining pressure that appear in the evolution equations are $\varepsilon \sim 0.005-0.5, \mathcal{S} \sim 0.5-2000$ and $\mathcal{A} \sim 10^{-8}-10^{-4}$ and are estimated using values given in [24]. The other dimensionless groups have ranges that are estimated using fluid properties given in $[55,56]$ such that realistic ranges are $\mathrm{Ca} \sim 10^{-5}-10^{-2}, K \sim 0-1, \Delta \sim 0-10^{-2}$, $\mathrm{Pe} \sim 10^{2}-10^{6}, \Theta \sim 0-1$ and $\mathrm{Ma} \sim 0-100$.

\section{RESULTS}

In this section, we describe the results of our parametric study. We begin by summarising the flow behaviour in the absence of particles-induced structural disjoining pressures in order to provide a baseline case against which these effects can be contrasted. A brief description of the numerical procedure used to perform the computations is provided first. 


\section{A. Numerical procedure}

The evolution equations are solved using a numerical procedure which employs the finitedifference approximation to discretise the spatial derivatives: centered-differences are used over the whole of the computational domain except for the end points where one-sided differences are utilised. In the majority of the cases described below, numerical solutions were obtained starting from the following initial condition

$$
\begin{aligned}
& h(x, 0)=2\left(1-x^{2}\right)+h_{\infty}, \quad \phi(x, 0)=\phi_{0} \quad \text { for } \quad|x| \leq 1 \\
& h(x, 0)=h_{\infty}, \quad \phi(x, 0)=0 \text { for } \quad|x|>1 .
\end{aligned}
$$

The initial condition for $c$ is then given by

$$
\begin{array}{ll}
c(x, 0) \equiv c_{0}=\phi_{0} /\left(1-\phi_{0}\right), & \text { for }|x| \leq 1 \\
c(x, 0)=0, & \phi(x, 0)=0 \text { for } \quad|x|>1 .
\end{array}
$$

The initial condition given by Eq. (27) corresponds to a droplet of constant curvature laden with nanoparticles of volume fraction $\phi_{0}$, released onto an ultra-thin film of uniform thickness, $h_{\infty}$ :

$$
h_{\infty}=\left(\frac{\Delta \mathcal{A}}{\Theta}\right)^{\frac{1}{3}} .
$$

The numerical solutions are subject to the following no-flux conditions

$$
\begin{aligned}
h_{x}(0, t)=h_{x x x}(0, t)=0, & c_{x}(0, t)=0, \\
h_{x}(L, t)=h_{x x x}(L, t)=0, & c_{x}(L, t)=0,
\end{aligned}
$$

where $L$ is the length of the computational domain. The thickness $h_{\infty}$ corresponds to an equilibrium, particle-free film, adsorbed from the atmosphere, which is stabilised by the presence of van der Waals forces. The thickness of $h_{\infty}$ increases with $\mathcal{A}$ and $\Delta$ and decreases with increasing superheat, $\Theta$. Thus the singularity at the advancing contact line of a drop spreading on a bare substrate is relieved by the presence of the equilibrium film. Note that this is not simply a device to remove the singularity, but a physical phenomenon created naturally by the interaction between evaporation and attractive van der Waals forces.

The value of $h_{\infty}=0.01$ is fixed for all the computations presented in this paper. Typically, 5000 grid points are used to carry out the computations for $0 \leq L \leq 5$. The fine grids used here overcome any minor regularity issues that are connected with the discontinuous gradient in the height field at the initial droplet edge; convergence is achieved upon mesh refinement. 
(a)

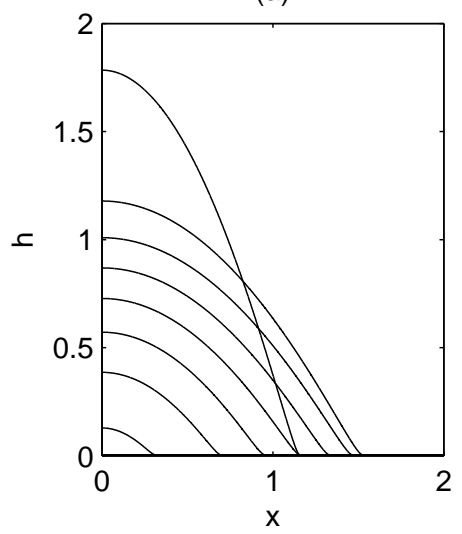

(d)

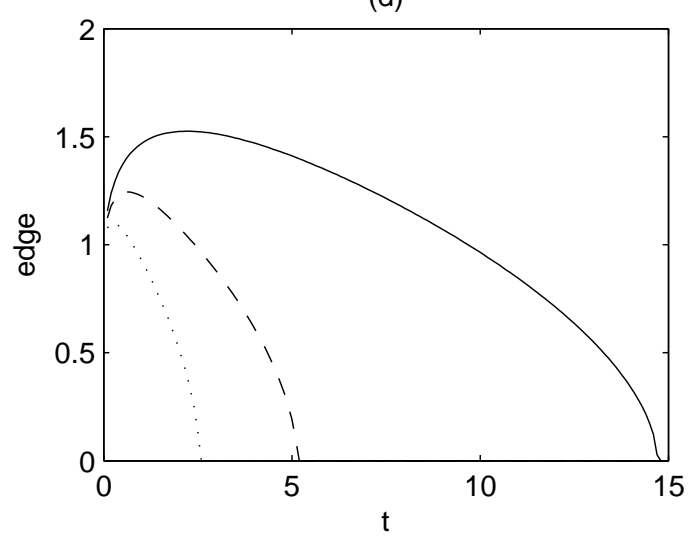

(b)

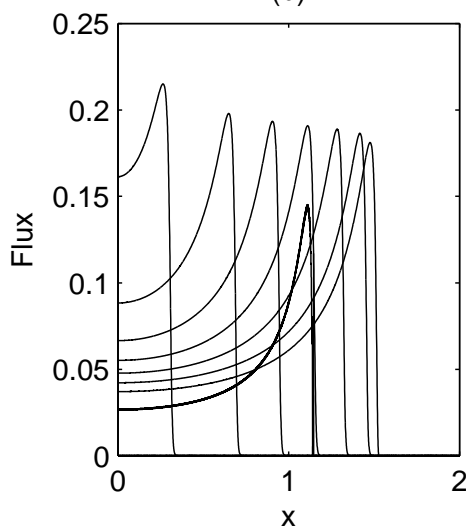

(c)

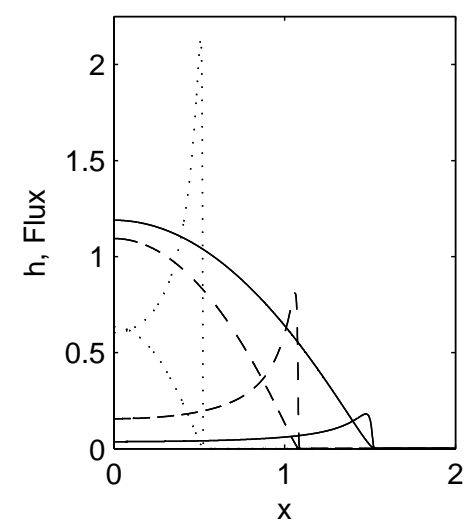

(e)

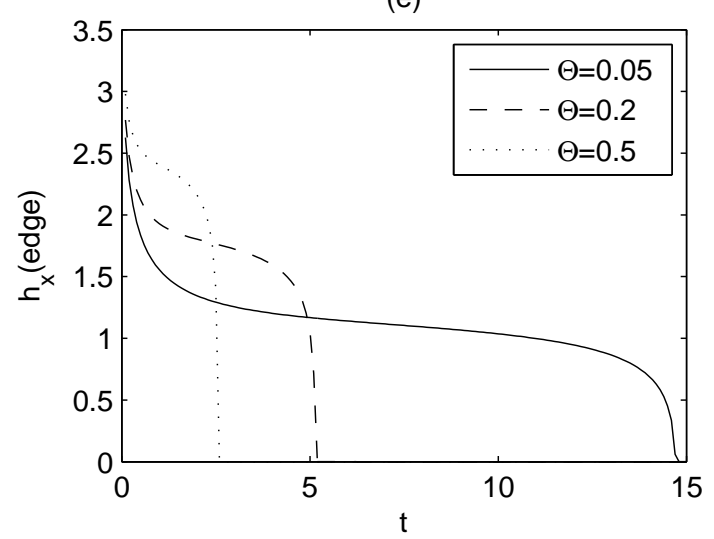

FIG. 1: Drop evolution in the presence of evaporation and absence of nanoparticles and Marangoni stresses with the following parameter values $K=0.2, \Delta=0.001, \mathcal{A}=10^{-6}$ and $\mathrm{Ma}=0$. Panels (a) and (b) show the spatio-temporal development of $h$ and $J$, respectively, at time intervals spaced by 2 dimensionless time units and starting from $t=0.1$. Panel (c) shows $h$ and $J$ at $t=5$ for different values of $\Theta$. Panels (d) and (e) show the effect of $\Theta$ on the temporal evolution of the droplet leading edge and the slope (microscopic contact angle) at this location, respectively.

\section{B. Negligible structural disjoining pressure}

We begin the presentation of our results by outlining briefly the system behaviour in the absence of nanoparticles [56]. This is done via numerical solution of Eq. (25) with $\mu=1$ and $\mathcal{S}=0$. A typical droplet evolution is shown in Fig. 1, in the absence of Marangoni stresses, $\mathrm{Ma}=0$, and with $\Theta=0.5, \mathrm{Ma}=20, K=0.2, \Delta=0.001, \mathrm{Pe}=10^{-4}$. It is clearly seen that the droplet undergoes spreading during the early stages of the flow, as shown in panels 
(a) $M a=100$

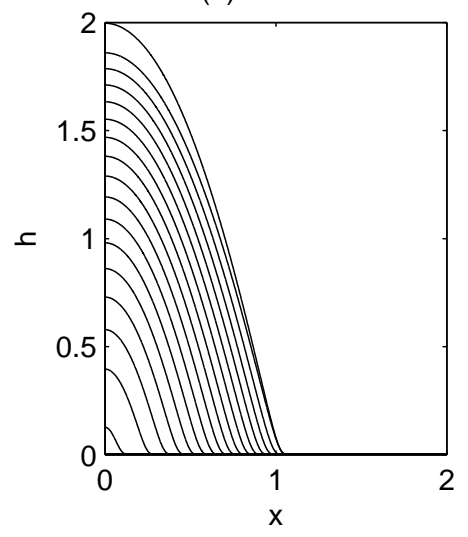

(d)

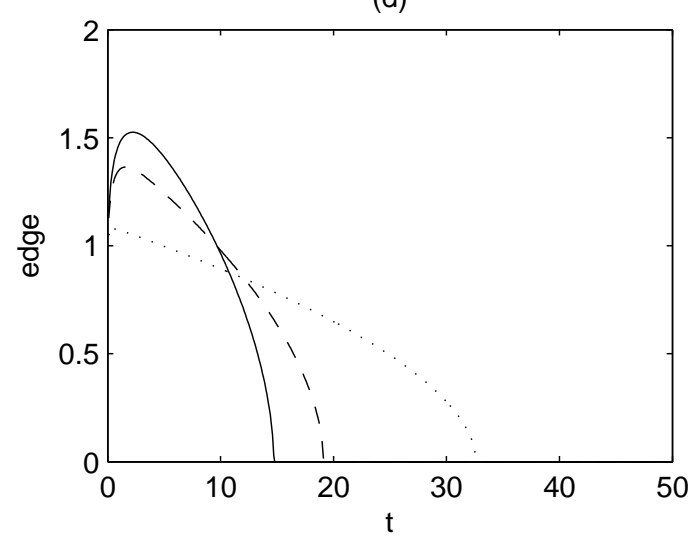

(b) $\mathrm{Ma}=100$

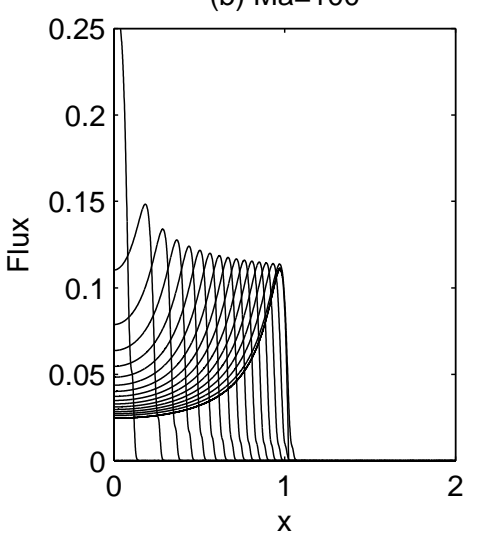

(c)

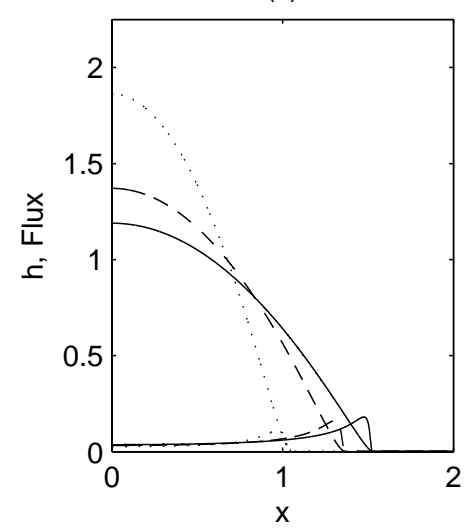

(e)

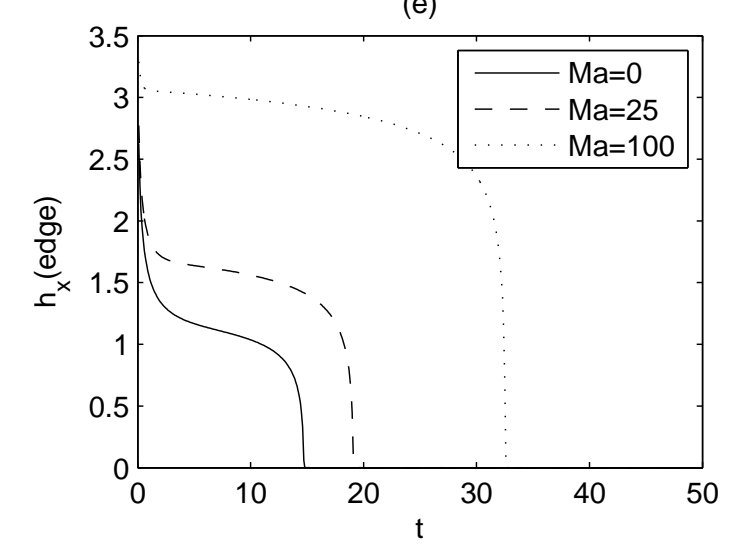

FIG. 2: Drop evolution in the presence of evaporation and Marangoni stresses, and absence of nanoparticles with the following parameter values $K=0.2, \Delta=0.001, \mathcal{A}=10^{-6}$ and $\Theta=0.05$. Panels (a) and (b) show the spatio-temporal development of $h$ and $J$, respectively, at time intervals spaced by 2 dimensionless time units and starting from $t=0.1$. Panel (c) shows $h$ and $J$ at $t=5$ for different values of Ma. Panels (d) and (e) show the effect of Ma on the temporal evolution of the droplet leading edge and the slope at this location, respectively.

(a) and (d) of Fig. 1 that depict the spatio-temporal evolution of $h$ and the droplet leading edge, respectively. It is also evident that mass loss takes place continuously by evaporation and this is accompanied by retraction of the leading edge until the droplet vanishes.

The evaporative flux exhibits a peak at the leading edge, as shown in Fig. 1(b), where the interfacial slope is largest and the thickness least. The amplitude of this peak rises with increasing superheat $\Theta$ as expected, which is demonstrated in Fig. 1(c). The 'contact angle' is also shown in Fig. 1(e), defined as the interfacial slope, $h_{x}$, evaluated at the 
leading edge, or droplet 'contact line'. Inspection of this figure reveals that the contact line dynamics exhibit three distinct stages corresponding to early, intermediate and late times. It is seen that the contact angle decreases rapidly during the early stage of the dynamics that corresponds to the time interval during which spreading occurs. In the intermediate stage, the contact angle decreases relatively slowly as the contact line undergoes retraction. The final stage is accompanied by a precipitous drop in the contact angle immediately before the droplet vanishes due to evaporation.

The presence of the three stages of the contact line dynamics appears to be robust for the various $\Theta$ values used. The duration of the intermediate stage decreases with increasing $\Theta$ as does the distance to which the droplet spreading prior to retraction, as shown in Fig. 1(d),(e). The addition of Marangoni stresses via the Marangoni parameter, Ma, acts to delay the eventual disappearance of the droplet by reducing the effective flux at its edge and retarding its initial spreading stage; this is shown in Fig. 2.

\section{Structural disjoining pressure}

In this section, we investigate the effect of structural disjoining pressure via numerical solution of Eqs. (25) and (26) with $\mathcal{S}>0$. In Fig. 3 we show the droplet evolution with $c_{0}=0.05$, and the rest of the parameters remaining unchanged from Fig. 1. As shown in panel (a) of Fig. 3, generated with $\mathcal{S}=1$, the behaviour of the droplet is similar to that in the absence of particles, albeit slightly retarded due to the viscosifying effect of the particles: the early stages of the dynamics are accompanied by spreading, which then gives way to evaporatively-driven leading edge retraction. The particles, as shown in Fig. 3(b,c), concentrate at the leading edge due to the high mass flux local to this region, and are left behind in the 'wake' of the receding edge.

Increasing the relative significance of the structural disjoining component by raising the value of $\mathcal{S}$ leads to the formation of 'steps' and 'terraces' at the droplet leading edge. As shown in Fig. 3(d,e), for a value of $\mathcal{S}=25$ a single step is formed, while, for $\mathcal{S}=200$, multiple steps are observed. These steps and terraces remain virtually stationary whilst the main droplet evaporates. The additional attraction of the structural disjoining pressure gives stable states where these effects balance the evaporation and fluid remains within the step. We cannot rule out the fact that these steps and terraces are unstable to spanwise 
perturbations. Indeed, following the work of [57] on receding nanoparticulate films, we expect these terraces to be vulnerable to a pattern-forming instability.

Also of interest is the effect of $\mathcal{S}$ on the temporal evolution of the droplet leading edge. As shown in Fig. 3(f), increasing $\mathcal{S}$ leads to a delay in droplet retraction. This is related to the formation of steps and terraces, which is typical of the dynamics at large $\mathcal{S}$. Moreover, it is evident from Fig. 3(f) that the evolution of the leading edge becomes weakly-dependent on variations in $\mathcal{S}$ for sufficiently large $\mathcal{S}$ values. We have also found that increasing the initial particle concentration, $c_{0}$, has a qualitatively similar effect to that of increasing $\mathcal{S}$. This is shown in Fig. 4, where by keeping $\mathcal{S}$ fixed and increasing $c_{0}$ from 0.05 to 0.5 , retardation of the leading edge retraction and the formation of more pronounced terraces are observed.

To emphasise that the main droplet evaporates leaving behind the steps and terraces we display in Fig. $3(\mathrm{~g})$ the position, $x_{90}(t)$, within which $90 \%$ of the droplet volume resides. In contrast to the edge position shown in panel (f) for, say, $\mathcal{S}=200$ this position recedes until $t \sim 16$ and is largely independent of $\mathcal{S}$. The structural component of disjoining pressure creates the steps and terraces, but the main droplet evaporates and recedes almost independently of the disjoining pressure. After $t \sim 16$ the cases that involve steps and terraces have a jump in $x_{90}$ to a constant position that is, as expected, $90 \%$ of the way along the step.

As noted in section III B, Marangoni stresses were found to retard the retraction of the leading edge. The effect of Marangoni stresses on the dynamics in the presence of particles are depicted in Fig. 5 for $\mathcal{S}=100$ and $c_{0}=0.05$. This plot shows that step-formation is most pronounced with $\mathrm{Ma}=0$, that is, in the absence of Marangoni stresses. The droplet then expands and recedes leaving the step of fluid behind, Fig. 5(c), although ultimately even the large Ma case leaves a step behind. Thus, at high values of $\mathcal{S}$ the disjoining pressure dominates, and the main droplet takes longer to evaporate. One notable feature is that the step continues to slowly grow in extent, this is due to the weak diffusion of particles characterised by the Peclet number. Not shown is the behaviour for low values of the disjoining pressure, $\mathcal{S}=1$, as then the effect of the particles is weak. Even with the droplet restrained by Marangoni stresses no terracing or step formation was detected.

The effect of varying the superheat, $\Theta$, on the dynamics has also been explored. In Fig. 6 we show that increasing $\Theta$ can overwhelm the effect of the structural disjoining pressure; one moves from a regime with step-formation to one in which the droplet retracts rapidly 
(a) $S=1, c_{0}=0.05$

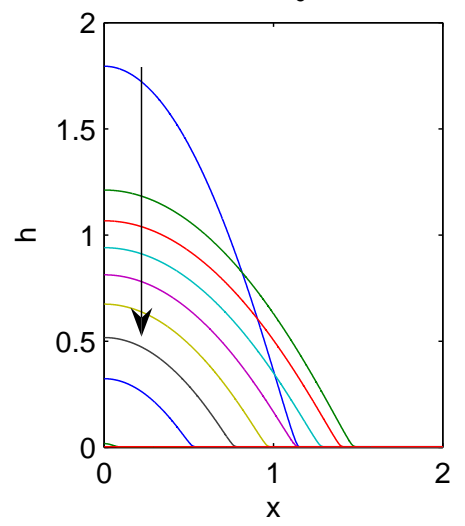

(d) $S=200, c_{0}=0.05$

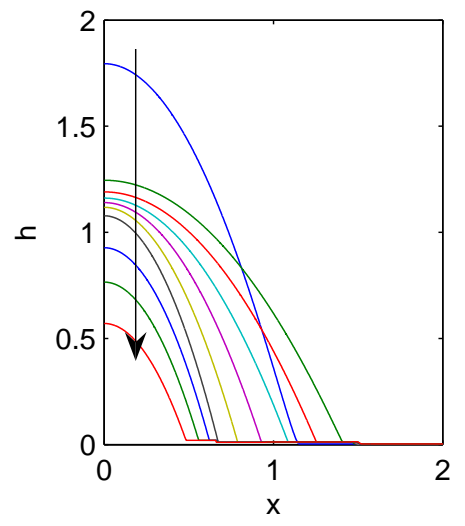

(b) $S=1, c_{0}=0.05$

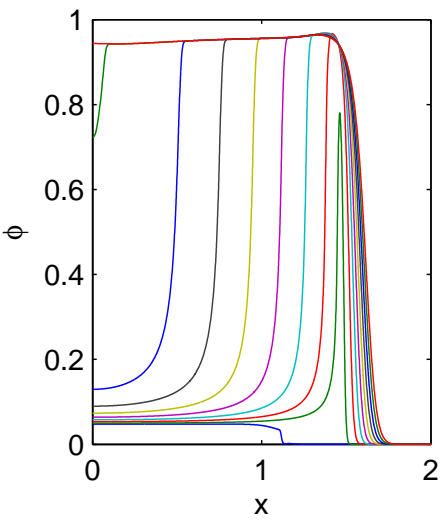

(e) Time $=100$

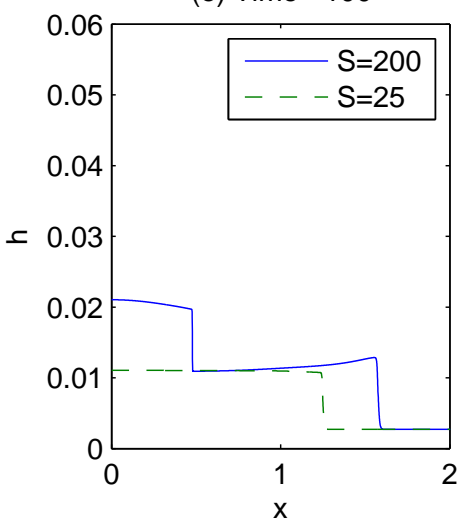

(c) Time $=5$

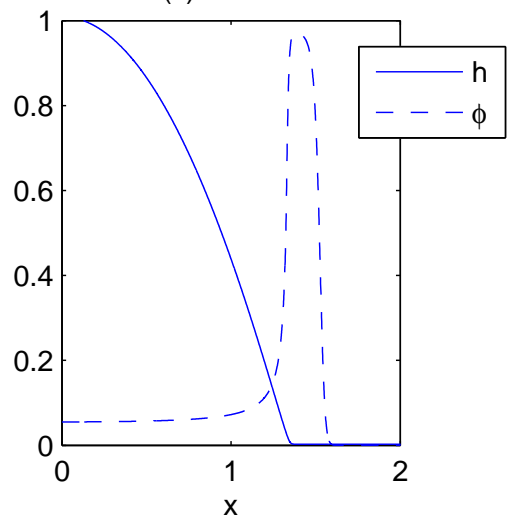

(f) Edge position

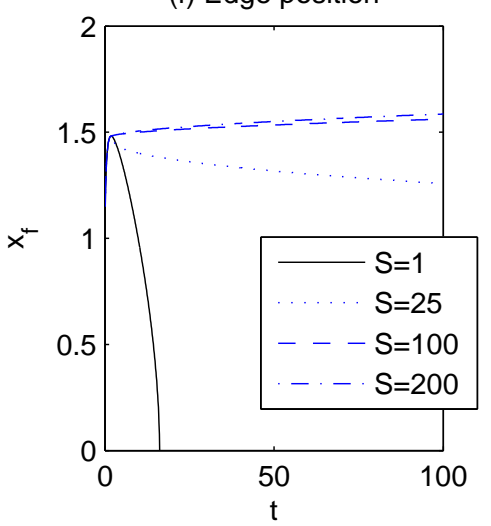

(g)

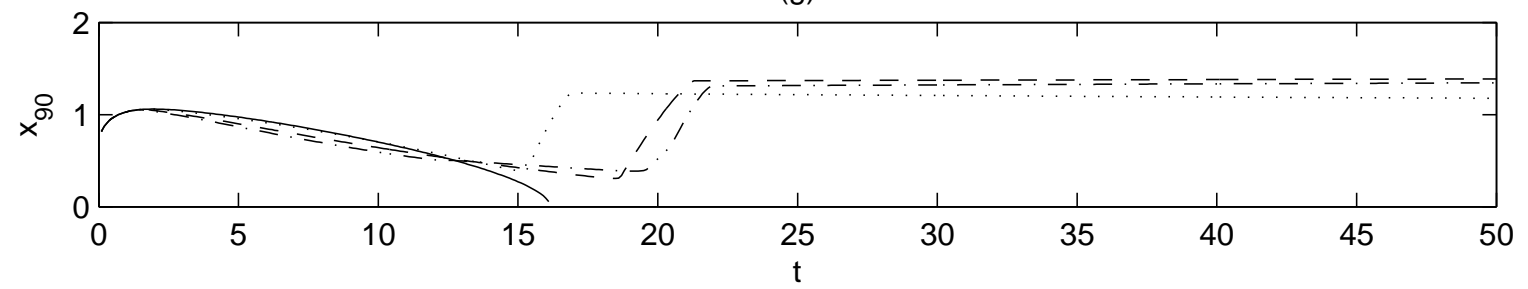

FIG. 3: Drop evolution in the presence of evaporation and nanoparticles. Panels (a) and (b) show the spatio-temporal development of $h$ and $\phi$, respectively, at time intervals spaced by 2 dimensionless time units and starting from $t=0.1$; (c) depicts $h$ and $\phi$ at $t=5$. In (a)-(c), $\mathcal{S}=1$ and $c_{0}=0.05$. Panel (d) is analogous to (a) except $\mathcal{S}=200$, and (e) presents enlarged versions of the leading edge region for $\mathcal{S}=25$ and $\mathcal{S}=200$ that exhibit 'step' and 'terrace' formation, respectively. The effect of varying $\mathcal{S}$ on the temporal evolution of the droplet leading edge is shown in (f). The rest of the parameter values remain unchanged from Fig. 1 with $\Theta=0.05$. The arrows in (a) and (d) indicate the direction of increasing time. Panel (g) shows the position, $x_{90}(t)$, within which $90 \%$ of the droplet volume resides. 
(a) Edge position

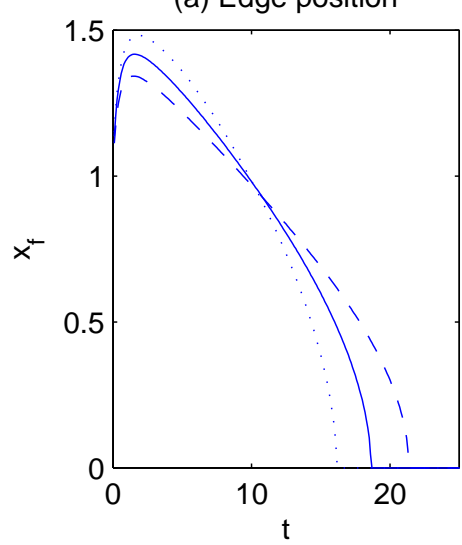

(b)

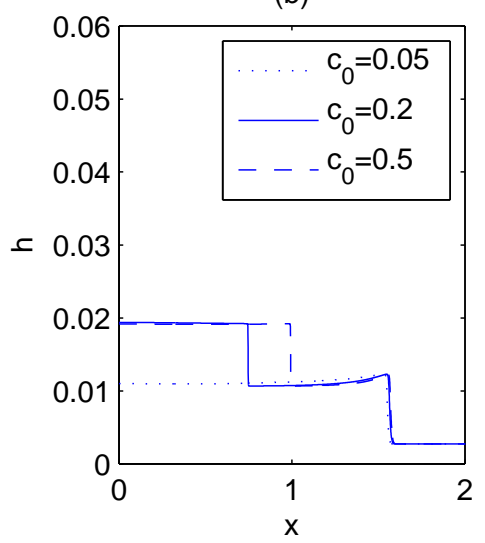

(c) Time $=5$

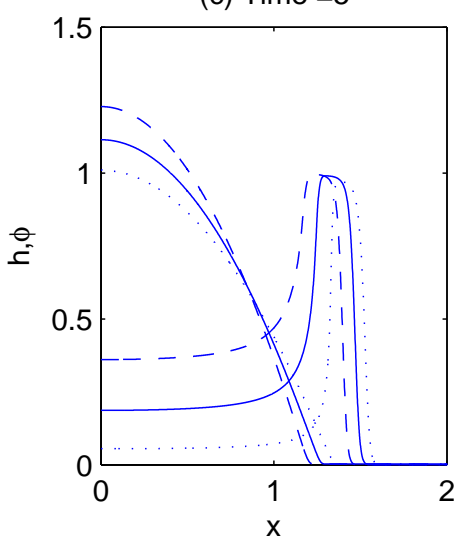

FIG. 4: The effect of altering the initial particle concentration, $c_{0}$, on the dynamics with $\Theta=0.05$, $K=0.2, \Delta=10^{-3}, \mathcal{A}=10^{-6}$. Panel (a) shows the temporal evolution of the droplet leading edge with $\mathcal{S}=1$ while (b) presents an enlarged version of this region for $\mathcal{S}=100, t=100$ and different values of $c_{0}$; (c) shows $h$ and $\phi$ at $t=5$ for the same parameters as those used to generate (b).

without forming steps. In panel (d) of Fig. 6 we show a position, $x_{90}(t)$, within which $90 \%$ of the fluid remains at a given time. For the case with the step, $\Theta=0.05$, this emphasises that the step remains locked in position, as shown by the plateau in panel (a), but that the main droplet evaporates and recedes. The sudden jump at $t \sim 15$ denotes the time at which only the step remains and $90 \%$ of the fluid remaining is then kept within a fixed domain.

\section{Long droplets and stability}

If the droplets are sufficiently long then it is possible that a Marangoni-driven instability will occur causing the droplet to break into a number of smaller droplets. We do not consider this scenario in detail as our main interest is in the advance/retraction of a single droplet and the effect of the structural disjoining pressure on the edge behaviour. It is important to note, however, that this alternative multiple breakup is a possibility and that a flat film or long droplet can suffer from instability whose origin can be easily identified using a linear stability analysis $[39,55,58]$.

A base state corresponding to an evaporating film of uniform thickness is obtained from 
(a) Edge position

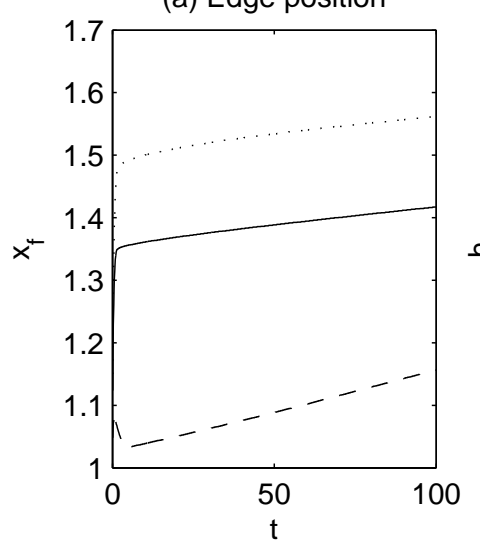

(b) Time $=5$

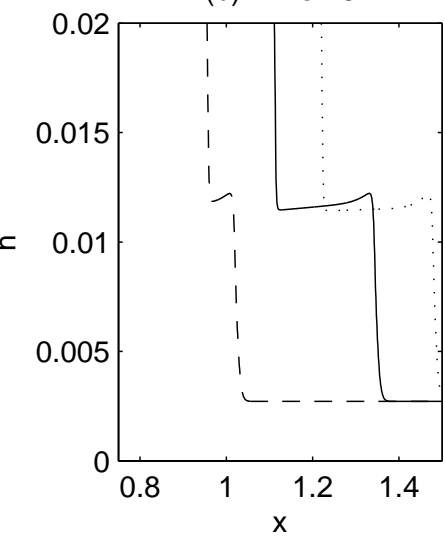

(c) Time $=5$

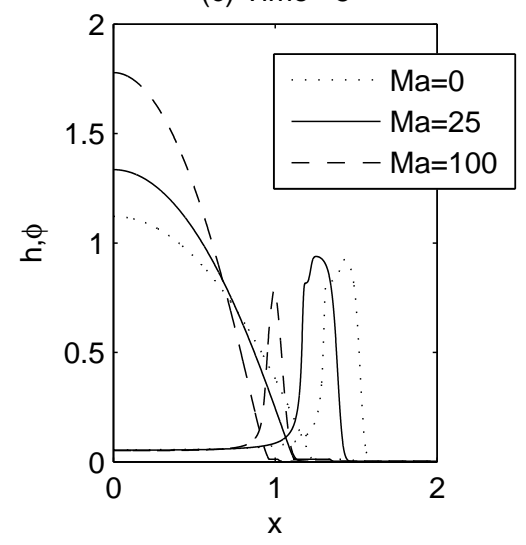

FIG. 5: The effect of altering Ma on the dynamics with $\Theta=0.05, K=0.2, \Delta=10^{-3}, \mathcal{A}=10^{-6}$ and $\mathcal{S}=100$; the initial concentration $c_{0}=0.05$. Panel (a) shows the temporal evolution of the droplet leading edge while (c) shows $h$ and $\phi$ at $t=5$ with (b) as an enlargement of the behaviour at the edge.
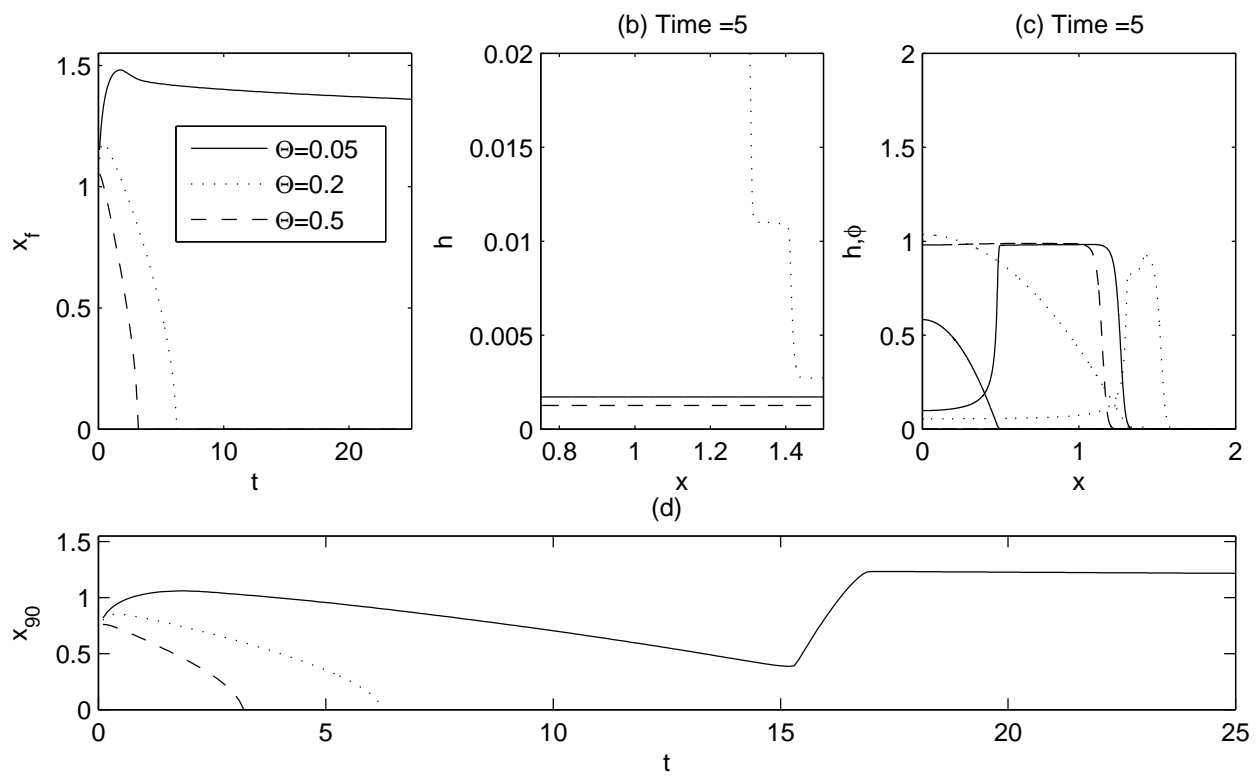

FIG. 6: The effect of varying superheat $\Theta$ on the dynamics with $K=0.2, \Delta=10^{-3}, \mathcal{A}=10^{-6}$ and $\mathcal{S}=1$ and initial concentration $c_{0}=0.05$. Panel (a) shows the temporal evolution of the droplet leading edge while (c) shows $h$ and $\phi$ at $t=5$ with (b) as an enlargement of the behaviour at the edge for $\Theta=0.05$. Panel (d) shows the position, $x_{90}(t)$, within which $90 \%$ of the fluid volume is contained. 
Eq. (25) by equating the unsteady term to $J$ (with $h_{x x}=0$ ):

$$
h_{*}\left(t_{*}\right)=-K+\sqrt{\left(K+h_{i}\right)^{2}-2 \Theta t_{*}},
$$

where $h_{i}$ is the initial thickness. Due to evaporation this base state is time-dependent, as expected. Substitution of the following normal-modes expansion $h\left(x, t ; t_{*}\right)=h_{*}\left(t_{*}\right)+$ $\hat{h} \exp (\omega t+i k x)$ into Eq. (25) and subsequent linearisation yields

$$
\begin{aligned}
\omega=k^{2}\left(\frac { M a } { 2 } h _ { * } ^ { 2 } \left[h_{*}\right.\right. & \left.\left.\left(\Delta k^{2}-\frac{\Theta}{K+h_{*}}\right) \frac{1}{K+h_{*}}+\frac{\Theta}{K+h_{*}}\right]-\frac{k^{2} h_{*}^{3}}{3}\right) \\
& -\frac{1}{K+h_{*}}\left(\Delta k^{2}-\frac{\Theta}{K+h_{*}}\right) .
\end{aligned}
$$

Here, $\hat{h} \ll h_{*}$ is the disturbance amplitude, $\omega$ and $k$ its growth rate and wavenumber. Note that in performing the above linear stability analysis, we have assumed implicitly that the perturbation time scale is much shorter than that associated with the base state. The quasi-steady-state approximation will therefore lead to an explicit, parametric dependence on $t_{*}$.

The effect of $\Delta, \Theta$, Ma and $K$ on the dispersion curves obtained from Eq. (32) is shown in Fig. 7 for the initial time; decreasing the film thickness maintains the same trends with these parameters, but with an increased growth rate. It is seen that an increase in the value of these parameters increases the growth rate of the majority of the $k$ interval (the exception being an increase in $K$ renders long-wave disturbances less unstable); this increase also shifts the wavenumber associated with the 'cut-off' and 'most dangerous' modes to larger wavenumbers.

We illustrate the effect of this linear instability on the nonlinear dynamics of an initially long drop, laden with particles but in the absence of structural disjoining pressure effects. In Fig. 8, we show the film evolution by solving Eqs. (25) and (26) with $\mathcal{S}=0$ starting from an initial condition given by

$$
\begin{aligned}
& h(x, 0)=1+h_{\infty}+\delta h, \quad \text { for } \quad|x|<2 \pi, \\
& h(x, 0)=\max \left(1-(x \mp 2 \pi)^{2}, 0\right)+h_{\infty} \quad \text { for } \quad|x| \geq 2 \pi,
\end{aligned}
$$

with the minus (plus) for positive (negative) $x ; \phi(x, 0)=0.1$ in the drop only. The droplet is a given a small amplitude perturbation of $\delta h=-0.01 \cos (\pi x)$ which is in the unstable range of wavenumbers for $\Theta=0.5, \mathrm{Ma}=20, K=0.2, \Delta=0.001, \mathcal{A}=10^{-6}$. As shown in Fig. 8, 
$\Delta$
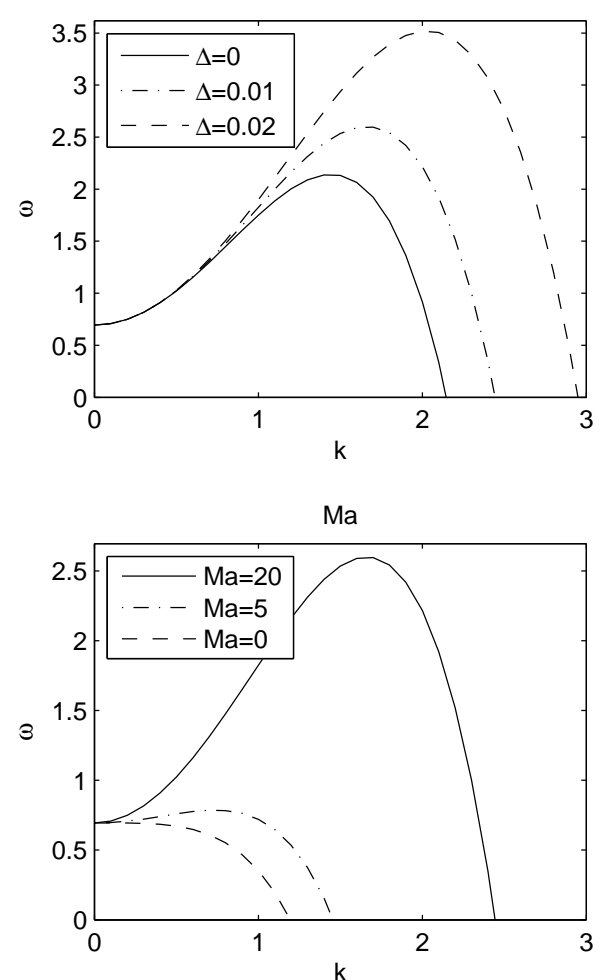

$\Theta$
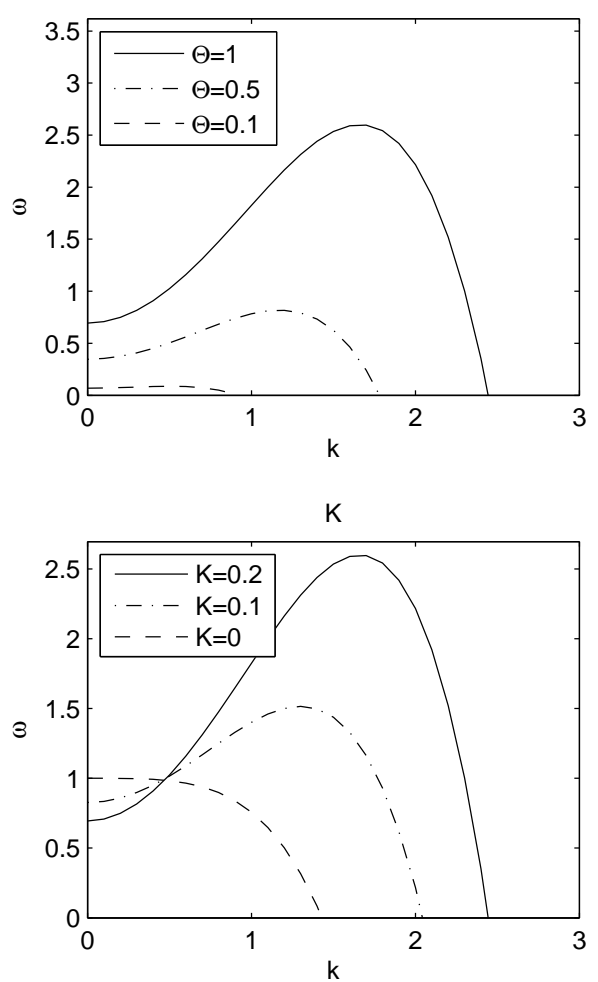

FIG. 7: The effect of $\Delta, \Theta$, Ma and $K$ on the dispersion curves generated from Eq. (32). Unless stated otherwise in the panel legends, the parameter values are $\Theta=1, \mathrm{Ma}=20, K=0.2, h_{*}=$ $1, \Delta=0.01$ and $t_{\star}=0$ with $h_{i}=1$.

the instability grows faster than the droplet retracts and thus it splits in two. Each smaller droplet then evaporates independently of the other. The instability is Marangoni-driven and the initial droplet thickness is large relative to the lengthscales over which disjoining pressure acts; the effect of the disjoining pressure is to prevent the droplet rupturing with the height field becoming zero. The volume fraction $\phi$ shows that the nanoparticles are deposited on the substrate as the droplet retracts.

\section{CONCLUDING REMARKS}

Our aim in this article is to generate a continuum model capable of producing pinned terraces and steps for evaporating droplets; these features are observed experimentally when droplets contain nanoparticles. The nanoparticles are taken into account within the model 
(a)

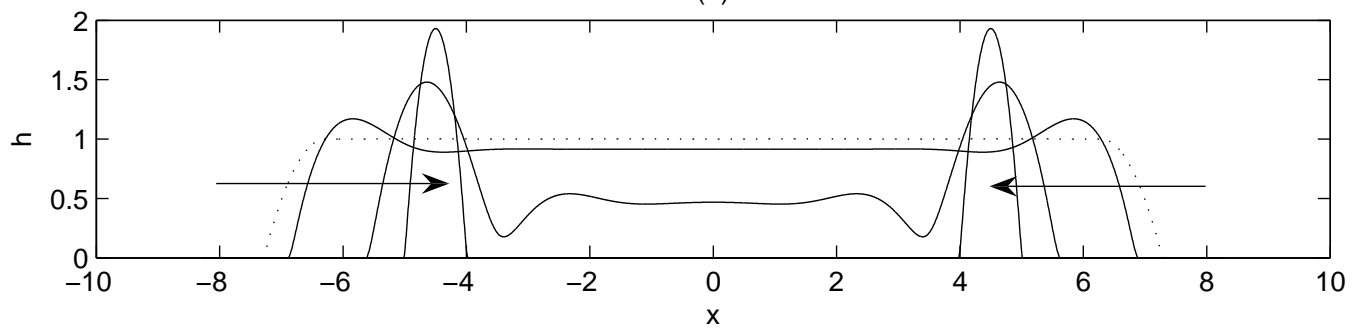

(b)

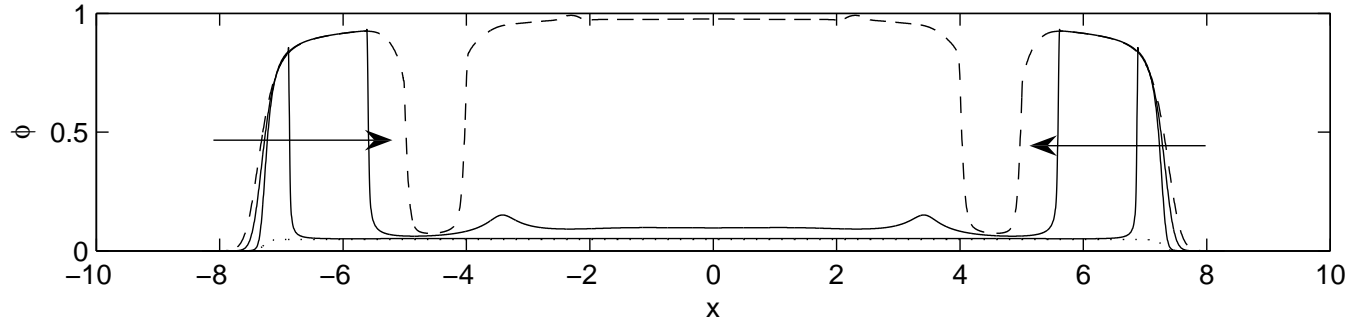

FIG. 8: The evolution of an initially long droplet starting from Eq. (33) with $\Theta=0.5, \mathrm{Ma}=$ $20, K=0.2, \Delta=0.001, c_{0}=0.05$. The height and particle volume fraction are shown in (a) and (b) for $t=0.2,1$ and 2 with the initial conditions shown as dotted lines and the arrows indicate increasing time. In panel (b) the particle fraction at $t=2$ is shown as a dashed line for clarity.

by the presence of structure disjoining pressure effects. The dynamics are modelled using lubrication theory which leads to the derivation of coupled equations for the position of the interface and particle concentration. The droplets are assumed to be sufficiently slender and vertical diffusion sufficiently rapid that these collectively act to erase vertical concentration gradients. The Kralchevsky-Denkov relation is employed in this work to model the presence of the structural disjoining pressure in the flow. Structural disjoining pressure models have oscillatory potentials and these lead to locally stable thickness that are the steps and terraces. Furthermore, we follow a similar approach to that used recently by Ajaev [30] and assume the presence of adsorbed ultra-thin stable films ahead of the droplet wherein evaporation is suppressed by the van der Waals disjoining pressure. We show through numerical solution of the evolution equations that increasing the relative significance of the structural disjoining pressure and/or increasing the initial particle concentration delays the retraction of the droplet leading edge, which normally accompanies droplet evaporation; it also leads to the formation of 'steps' and 'terraces' at the leading edge. Increasing the degree of superheat, on the other hand, was found to promote retraction and suppress step and terrace-formation. 
Marangoni stresses act to dramatically delay evaporation via reducing the flux at the main droplet edge, and can also lead to an instability that breaks up longer and thinner droplets on a timescale more rapid than the edge retraction created by evaporation.

\section{APPENDIX A: FORMULATION DETAILS}

At the interface, we impose a condition of mass conservation:

$$
J=\rho_{l}\left(\mathbf{u}-\mathbf{u}_{s}\right) \cdot \mathbf{n}=\rho_{v}\left(\mathbf{u}_{v}-\mathbf{u}_{s}\right) \cdot \mathbf{n}
$$

where $\rho_{l}$ and $\rho_{v}$ denote the liquid and vapour densities, respectively; $\mathbf{u}_{v}$ is the vapour velocity and $\mathbf{u}_{s}$ is the velocity of the interface; $\mathbf{n}$ is the outward pointing normal to the interface. We also impose a kinematic boundary condition:

$$
h_{t}+u_{s} h_{x}=w_{s}
$$

Elimination of $u_{s} h_{x}-w_{s}$ from Eqs. (A1) and (A2) yields the kinematic condition in Eq. (2), which is expressed in terms of the evaporative flux $J$

$$
h_{t}+u h_{x}-w+\frac{J}{\rho_{l}}=0 .
$$

The concentration, $c$, corresponds to the mass of particles per unit volume of fluid: $c=m / V_{f}$. Here, $m=\rho_{p} V_{p}$ where $\rho_{p}$ and $V_{p}$ are the particle density and volume, respectively. The volume fraction of particles, $\phi$, is the volume of particles divided by the total volume: $\phi=V_{p} /\left(V_{f}+V_{p}\right)$. Elimination of $V_{p}$ yields

$$
\phi=\frac{c / \rho_{p}}{1+c / \rho_{p}} .
$$

However, crucially this ignores the fact that the particles have a maximal packing fraction, $\phi_{m}$, and that $p h i$ is inevitably bounded above by this value $(\sim 0.664)$ rather than unity. Thus we scale (A4) on $\phi_{m}$ to build in this extra piece of physics so

$$
\phi=\phi_{m} \frac{c / \rho_{p}}{1+c / \rho_{p}}
$$

[1] J. N. Israelachvili, Intermolecular and Surface Forces (Academic Press, San Diego, CA, 1992). 
[2] N. V. Churaev, Adv. Colloids Interface Sci. 103, 197 (2003).

[3] S. Asakura and F. Oosawa, J. Chem. Phys. 22, 1255 (1954).

[4] R. Kjellander and S. Sarman, Chem. Phys. Lett. 149, 102 (1988).

[5] P. Attard and J. L. Parker, J. Phys. Chem. 96, 5086 (1992).

[6] P. A. Kralchevsky and N. D. Denkov, Chem. Phys. Lett. 240, 385 (1995).

[7] B. Götzelmann and S. Dietrich, Phys. Rev. E 57, 6785 (1998).

[8] A. Trokhymchuk, D. Henderson, A. Nikolov, and D. T. Wasan, Langmuir 17, 4940 (2001).

[9] A. D. Nikolov and D. T. Wasan, J. Colloid Interface Sci. 133, 1 (1989).

[10] A. D. Nikolov, P. A. Kralchevsky, I. B. Ivanov, and D. T. Wasan, J. Colloid Interface Sci. 133, 13 (1989).

[11] A. D. Nikolov and D. T. Wasan and N. D. Denkov and P. A. Kralchevsky and I. B. Ivanov, Prog. Colloid Polym. Sci. 82, 87 (1990).

[12] D. T. Wasan, A. D. Nikolov, P. A. Kralchevsky, and I. B. Ivanov, Colloids Surf. 67, 139 (1992).

[13] V. Bergeron and C. J. Radke, Langmuir 8, 3020 (1992).

[14] V. Bergeron, A. J. Jimenez-Laguna, and C. J. Radke, Langmuir 8, 3027 (1992).

[15] X. L. Chu, A. D. Nikolov, and D. T. Wasan, J. Chem. Phys. 103, 6653 (1995).

[16] X. L. Chu, A. D. Nikolov, and D. T. Wasan, J. Chem. Phys. 105, 4892 (1996).

[17] D. Henderson, S. Sokolowski, and D. T. Wasan, J. Stat. Phys. 89, 233 (1997).

[18] S. Manne and G. Warr, eds., Supramolecular structures in confined geometries (American Chemical Society, Washington, DC, 1999).

[19] F. Heslot, N. Fraysse, and A. M. Cazabat, Nature 338, 640 (1989).

[20] S. Betelu, B. M. Law, and C. C. Huang, Phys. Rev. E 59, 6699 (1999).

[21] D. T. Wasan and A. D. Nikolov, Nature 423, 156 (2003).

[22] R. L. Kao, D. T. Wasan, A. D. Nikolov, and D. A. Edwards, Colloids Surf. 34, 389 (1989).

[23] A. Chengara, A. D. Nikolov, D. T. Wasan, A. Trokhymchuk, and D. Henderson, J. Colloid Interface Sci. 280, 192 (2004).

[24] O. K. Matar, R. V. Craster, and K. Sefiane, Phys. Rev. E 76, 056315 (2007).

[25] D. M. Anderson and S. H. Davis, Phys. Fluids 7, 248 (1995).

[26] A. Oron, S. H. Davis, and S. G. Bankoff, Rev. Mod. Phys. 69, 931 (1997).

[27] P. Kavehpour, B. Ovryn, and G. H. McKinley, Colloids Surf. A 206, 409 (2002). 
[28] M. Cachile, M. Schneemilch, A. Hamraoui, and A. M. Cazabat, Adv. Colloid Interface Sci. 96, 59 (2002).

[29] C. Poulard, O. Benichou, and A. M. Cazabat, Langmuir 19, 8828 (2003).

[30] V. S. Ajaev, J. Fluid Mech. 528, 279 (2005).

[31] H. Hu and R. G. Larson, Langmuir 21, 3963 (2005).

[32] H. Hu and R. G. Larson, Langmuir 21, 3972 (2005).

[33] S. David, K. Sefiane, and L. Tadrist, Colloids Surf. A 298, 108 (2007).

[34] E. Adachi, A. S. Dimitrov, and K. Nagayama, Langmuir 11, 1057 (1995).

[35] R. D. Deegan, O. Bakajin, T. F. Dupont, G. Huber, S. R. Nagel, and T. A. Witten, Nature 389, 827 (1997).

[36] R. D. Deegan, O. Bakakin, T. F. Dupont, G. Huber, S. R. Nagel, and T. A. Witten, Phys. Rev. E 62, 756 (2000).

[37] L. Shmuylovich, A. Q. Shen, and H. A. Stone, Langmuir 18, 3441 (2002).

[38] P. Takhistov and H.-C. Chang, Ind. Eng. Chem. Res. 41, 6256 (2002).

[39] M. R. E. Warner, R. V. Craster, and O. K. Matar, J. Colloid Interface Sci. 267, 92 (2003).

[40] E. Rabani, D. R. Reichman, P. L. Geissler, and L. E. Brus, Nature 426, 271 (2003).

[41] E. Rio, A. Daerr, F. Lequeux, and L. Limat, Langmuir 22, 3186 (2006).

[42] E. Pauliac-Vaujour, A. Stannard, C. P. Martin, M. O. Blunt, I. Nottinger, P. J. Moriarty, I. Vancea, and U. Thiele, Phys. Rev. Lett. p. 176102 (2008).

[43] L. V. Govor, G. Reiter, J. Parisi, and G. H. Bauer, Phys. Rev. E 69, 061609 (2004).

[44] L. V. Govor, G. Reiter, G. H. Bauer, and J. Parisi, Appl. Phys. Lett. 84, 4774 (2004).

[45] L. V. Govor, J. Parisi, G. H. Bauer, and G. Reiter, Phys. Rev. E 71, 051603 (2005).

[46] L. V. Govor, G. Reiter, G. H. Bauer, and J. Parisi, Phys. Rev. E 74, 061603 (2006).

[47] E. Bormashenko, R. Pogreb, O. Stanevsky, Y. Bormashenko, T. Stein, and O. Gengelman, Langmuir 21, 9604 (2005).

[48] E. Bormashenko, R. Pogreb, O. Stanevsky, Y. Bormashenko, T. Stein, V. Z. Gaisin, R. Cohen, and O. V. Gendelman, Macromol. Mater. Eng. 290, 114 (2005).

[49] C. Monteux, Y. Elmaallem, T. Narita, and F. Lequeux, Europhys. Lett. 83, 34005 (2008).

[50] R. V. Craster and O. K. Matar, Dynamics and stability of thin liquid films, Rev. Mod. Phys., in press.

[51] S. Moosman and G. M. Homsy, J. Colloid Interface Sci. 73, 212 (1980). 
[52] V. S. Ajaev and G. M. Homsy, J. Colloid Interface Sci. 240, 259 (2001).

[53] P. Attard, D. R. Bérard, C. P. Ursenbach, and G. N. Patey, Phys. Rev. A 44, 8224 (1991).

[54] O. E. Jensen and J. B. Grotberg, Phys. Fluids A 5, 58 (1993).

[55] J. P. Burelbach, S. G. Bankoff, and S. H. Davis, J. Fluid Mech. 195, 463 (1988).

[56] V. S. Ajaev, Phys. Rev. E 72, 031605 (2005).

[57] A. V. Lyushnin, A. A. Golovin, and L. M. Pismen, Phys. Rev. E 65, 021602 (2002).

[58] A. Oron, Phys. Fluids 12, 1633 (2000). 